\title{
Mechanism of Chain Collapse of Strongly Charged Polyelectrolytes
}

\author{
Anvy Moly Tom, ${ }^{1, *}$ Satyavani Vemparala, ${ }^{1, \dagger}$ R. Rajesh, ${ }^{1, \$}$ and Nikolai V. Brilliantov ${ }^{2, \$}$ \\ ${ }^{1}$ The Institute of Mathematical Sciences, C.I.T. Campus, Taramani, Chennai 600113, India \\ ${ }^{2}$ Department of Mathematics, University of Leicester, Leicester LE1 7RH, United Kingdom
}

(Received 19 May 2016; published 26 September 2016)

\begin{abstract}
We perform extensive molecular dynamics simulations of a charged polymer in a good solvent in the regime where the chain is collapsed. We analyze the dependence of the gyration radius $R_{g}$ on the reduced Bjerrum length $\ell_{B}$ and find two different regimes. In the first one, called a weak electrostatic regime, $R_{g} \sim \ell_{B}^{-1 / 2}$, which is consistent only with the predictions of the counterion-fluctuation theory. In the second one, called a strong electrostatic regime, we find $R_{g} \sim \ell_{B}^{-1 / 5}$. To explain the novel regime we modify the counterion-fluctuation theory.
\end{abstract}

DOI: 10.1103/PhysRevLett.117.147801

Introduction.-The conformational states of a flexible neutral polymer in different solvents are well known. It is extended in a good solvent due to favorable excluded volume interactions with the solvent molecules and collapses into a compact globule in a bad solvent [1-3]. In contrast, a flexible polyelectrolyte (PE) - charged polymer in the presence of counterions-undergoes an extended to collapsed transition in both good and bad solvents. Unlike neutral polymers, the conformations of a PE depend not only on the solvent quality, but also crucially on the interplay between electrostatic energy and translational entropy of counterions $[4,5]$. The strength of the electrostatic interactions depends on the charge density along the PE, which is quantified by the dimensionless Bjerrum length $\ell_{B}$. For small charge density, counterions are dispersed away from the PE, and the chain is in an extended necklace conformation when in a good or theta solvent [3], and is collapsed into a compact globule in a bad solvent $[3,6]$. With increasing charge density, the PE attains an extended conformation, regardless of the solvent quality and counterions begin to condense onto the PE, renormalizing its charge density $[3,7,8]$. Further increase of the PE charge density results in an effective attraction between similarly charged monomers of the PE and it collapses into a globule conformation, independent of the solvent quality [4-6,9-14].

The compaction of a PE chain into a globular conformation is of great biological importance. For instance, biological PEs like RNA or DNA are densely packed in cells and viruses [15-17] which are orders of magnitude smaller than the contour length of the PE, requiring it to be highly compacted $[18,19]$. Furthermore, the effective interactions driving the collapse of a single PE chain are closely connected to those resulting in aggregation of rigid PEs $[20,21]$. Common biological polymers like DNA, actin, and microtubules are examples of rigid PEs whose aggregates play a key role in functions like cell scaffolding, making it vital to understand the nature of attractive forces between similar charges [22]. Although for compaction or folding of biological PEs some specific mechanisms may be important, the mechanism of electrostatic collapse, as for a simple homopolymer PE chain, is still essential.

To describe the counterintuitive phenomenon of PE collapse in a good solvent, several competing theories have been proposed [4,19,23-27]. The first theory is based on modeling the collapsed conformation as an amorphous ionic solid [19]. For large charge density of the PE and in the presence of multivalent counterions the free energy of the solid is smaller than that of the extended PE, driving the chain collapse. This theory, however, does not predict any dependence of the gyration radius $R_{g}$ on $\ell_{B}$. In the second group of theories, it is assumed that condensed counterions and the PE monomers form dipoles [23,25-27]. The dipoles freely rotate yielding, on average, an attractive interaction between the segments of the chain; this leads to collapse of a PE even in a good solvent. For a highly charged flexible PE in a salt-free solution, this theory predicts that the radius of gyration of the collapsed conformation scales as $R_{g} \sim N^{1 / 3}\left|\ell_{B}^{2}-c B\right|^{-1 / 3}$, where $B$ is the second virial coefficient, $N$ is the number of chain monomers, and $c$ is a dimensional constant that depends on the details of the system [28]. This dependence is predicted for both $\operatorname{good}[23,26,27]$ and bad $[23,27]$ solvents. For theta solvent with $B=0$ [3], a simpler scaling, $R_{g} \sim \ell_{B}^{-2 / 3} N^{1 / 3}$ is obtained [23].

Finally, the third theory, referred to as counterionfluctuation theory, argues that the collapse of a PE is due to negative pressure arising from fluctuations in the density of condensed counterions, which move freely within a PE globule [4]. Such a physical picture of the condensed counterion motion agrees with the recent results of molecular dynamics (MD) simulations [14]. The counterion-fluctuation theory, when restricted to the second virial coefficient, predicts that in a good solvent $R_{g} \sim$ $\ell_{B}^{-1 / 2} N^{1 / 3}$. Note that all mechanisms discussed above imply a collapsed phase, $R_{g} \sim N^{1 / 3}$ for large charge density, but different dependence of $R_{g}$ on $\ell_{B}$. 
Because of a great significance for applications, especially for nanomedicine and biotechnology, it is vital to have an appropriate theory of the interactions that drive the collapse of a PE. In this Letter, we report the results of extensive MD simulations, exploring the collapsed conformation of a single flexible PE chain in a good solvent. Two regimes in the dependence $R_{g}\left(\ell_{B}\right)$ have been numerically revealed: The one, consistent with the counterion fluctuation theory, $R_{g} \sim \ell_{B}^{-1 / 2}$ [4], and the new regime, $R_{g} \sim \ell_{B}^{-1 / 5}$, which we explain modifying the above theory.

$M D$ simulations.-We model a flexible PE chain as $N$ monomers of charge $e(e>0$ is the elementary charge) connected by harmonic springs of energy,

$$
U_{\text {bond }}(r)=\frac{1}{2} k(r-a)^{2},
$$

where $k$ is the spring constant, $a$ is the equilibrium bond length, and $r$ is the distance between the bonded monomers. The chain and $N_{c}=N / Z$ neutralizing counterions, each of charge $-Z e$, with $Z=1,2,3$ being the valency, are placed in a box of linear size $L$. Pairs of all nonbonded particles (counterions and monomers) separated by a distance $r$ interact through the 6-12 Lennard Jones potential cutoff at $r_{c}$ :

$$
U_{\mathrm{LJ}}(r)=4 \epsilon\left[(\sigma / r)^{12}-(\sigma / r)^{6}\right] .
$$

The values of $\epsilon$ and $r_{c}$ are varied depending on the system being simulated. The electrostatic energy between charges $q_{i}$ and $q_{j}$ separated by $r_{i j}$ is

$$
U_{c}\left(r_{i j}\right)=\frac{q_{i} q_{j}}{\varepsilon r_{i j}},
$$

where $\varepsilon$ is the dielectric permittivity of the solution. The charge density along the PE chain is parametrized by the dimensionless Bjerrum length $\ell_{B}$ [3]:

$$
\ell_{B}=\frac{1}{a} \frac{e^{2}}{\left(\varepsilon k_{B} T\right)}=\frac{\beta e^{2}}{\varepsilon a},
$$

where $k_{B}$ is the Boltzmann constant, $T$ is temperature, and $\beta=\left(k_{B} T\right)^{-1}$. Larger $\ell_{B}$ corresponds to higher charge density of the PE. In the simulations, we use $a=1.12 \sigma, k=500.0 \epsilon_{0} / \sigma^{2}, L=370 \sigma$ and the temperature, $k_{B} T / \epsilon_{0}=1$, is maintained through a Nosé-Hoover thermostat. The long-ranged Coulomb interactions are evaluated using the particle-particle-particle-mesh (PPPM) technique, e.g., Refs. [6,20].

We now discuss the results from MD simulations of a single PE in a good solvent with purely repulsive LJ interactions between all nonbonded pairs of monomers and counterions. The cutoff of the LJ interaction is set at $r_{c}=\sigma$, and the energy constant is $\epsilon=\epsilon_{0}$. We simulate the system for values of $\ell_{B}$ where the equilibrium configuration of a PE is a collapsed state with $R_{g} \sim N^{1 / 3}$. The variation of the radius of gyration $R_{g}$ with $\ell_{B}$ in the globular regime is shown in Fig. 1. It can be seen from Fig. 1 that for $\ell_{B}<\ell_{B}^{*}(Z)$ the observed dependence, $R_{g} \propto \ell_{B}^{-1 / 2} N^{1 / 3}$, is consistent with the predictions of the counterion-fluctuation theory [4]. For $\ell_{B}>\ell_{B}^{*}(Z)$, we find a crossover to a different scaling, $R_{g} \propto \ell_{B}^{-1 / 5} N^{1 / 3}$, which is not predicted by any of the existing theories. The two regimes of $\ell_{B}<\ell_{B}^{*}(Z)$ and $\ell_{B}>\ell_{B}^{*}(Z)$ will be referred to as weak and strong electrostatic regimes, respectively.

Typical snapshots of the system with monovalent counterions in weak and strong electrostatic regimes are shown in Fig. 2, which demonstrates that the PE is much more compact in the strong electrostatic regime. Associated number density profile of counterions measured from the center of mass of the collapsed PE is also shown in Fig. 2. It can be seen that the profile has a broader tail in the weak
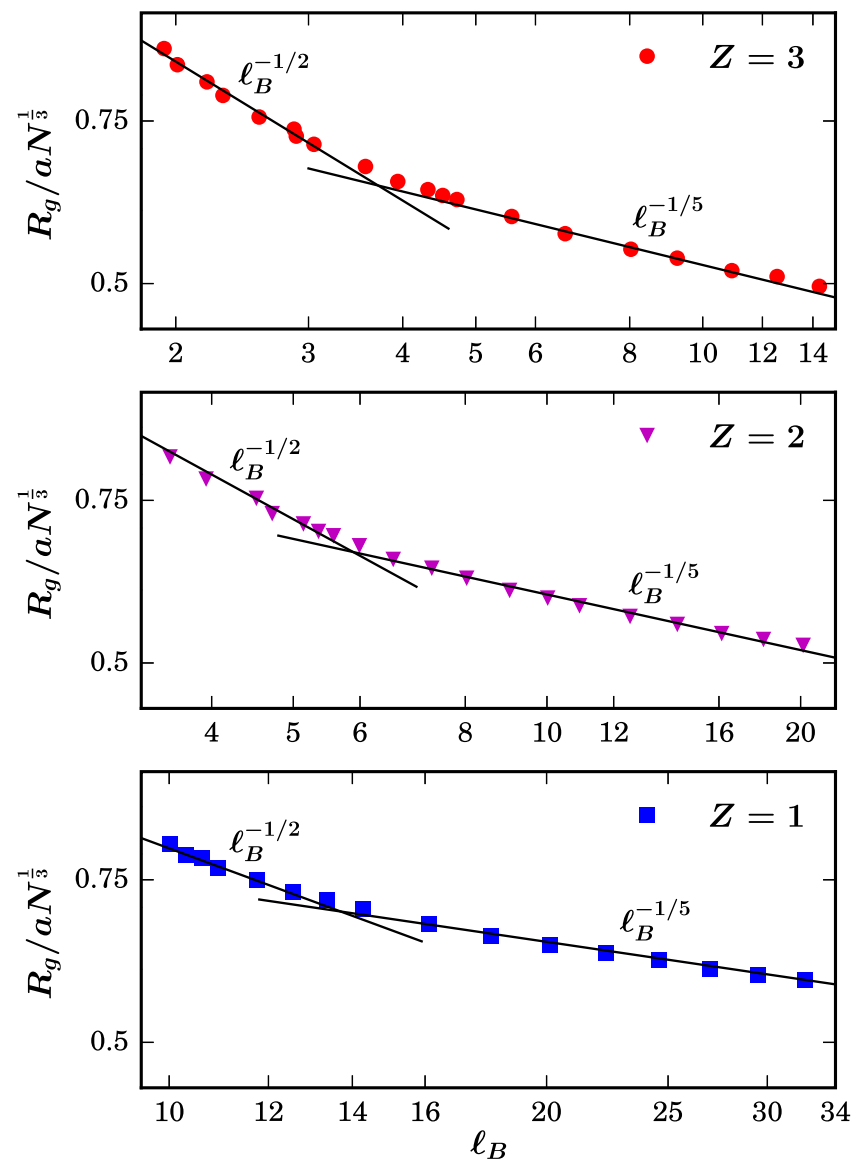

FIG. 1. Variation of the gyration radius $R_{g}$ of a PE chain with the reduced Bjerrum length $\ell_{B}$ for different valencies of counterions. The chain length is $N=204$. The two power laws intersect at $R_{g} / a N^{1 / 3} \approx 0.63(Z=3), 0.66(Z=2)$, and $0.69(Z=1)$ with the corresponding crossover values $\ell_{B}^{*}(Z) \approx 3.71(Z=3), 5.58$ $(Z=2)$, and $13.70(Z=1)$. 

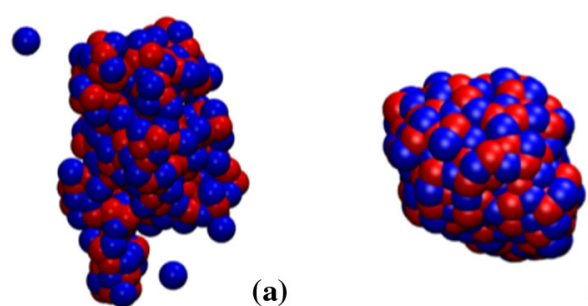

(a)

(b)

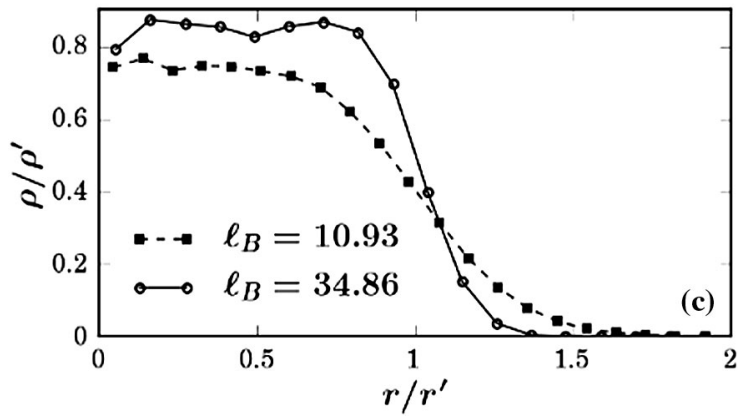

FIG. 2. Snapshots of collapsed PE in (a) weak electrostatic regime, $Z=1, \ell_{B}=10.93$ (left) and (b) strong electrostatic regime, $Z=1, \ell_{B}=34.86$ (right). (c) The corresponding radial number density profile $\rho$ of the counterions, where $r$ is the distance of the counterion from the center of mass of the chain. $r^{\prime}$ is the distance at which the density is $50 \%$ of the density at $r=0$ and $\rho^{\prime}=N / V^{\prime}$, where $V^{\prime}=\frac{4}{3} \pi r^{\prime 3}$.

electrostatic regime, suggesting that the counterions are more loosely bound.

We have verified that the exponents and associated features seen in Fig. 1 are robust and independent on the details of the interaction by simulating two other good solvent conditions (see the Supplemental Material [29]): (i) $\mathrm{LJ}$ interactions being attractive $\left(r_{c}=2.5 \sigma, \epsilon=0.25 \epsilon_{0}\right)$ for monomer-monomer pairs and purely repulsive $\left(r_{c}=1.0 \sigma, \epsilon=\epsilon_{0}\right)$ for all other pairs and (ii) $\mathrm{PE}$ in the presence of explicit solvent molecules with attractive interactions between monomers and solvent $\left(r_{c}=2.5 \sigma\right.$, $\epsilon=\epsilon_{0}$ ) and repulsive for all other pairs. We also confirm that the results are independent of the length of the chain for all values of $\ell_{B}$ that we have simulated (see the Supplemental Material [29]).

The dependence of $R_{g}$ on $\ell_{B}$ in the weak electrostatic regime supports the basic mechanism of the counterionfluctuation theory as described in Ref. [4], where the PE free energy was truncated at the second virial coefficient. We now re-examine this theory to explain the dependence $R_{g} \propto \ell_{B}^{-1 / 5} N^{1 / 3}$ in the strong electrostatic regime by including more terms in the virial expansion of the PE free energy: namely, we use the simplest generalization of the counterion-fluctuation theory [4], including the third virial coefficient $C$.

Theory.-The free energy of the system as a function of the radius of gyration $R_{g}$ of a PE chain can be written as $[3,4,31]$

$$
F\left(R_{g}\right)=F_{\text {id.ch }}\left(R_{g}\right)+F_{\text {vol }}\left(R_{g}\right)+F_{\text {el }}\left(R_{g}\right) .
$$

Here $F_{\text {id.ch }}\left(R_{g}\right)$ is the entropic part of the free energy corresponding to the ideal chain $[3,4,32]$,

$$
\beta F_{\text {id.ch }} \simeq \frac{9}{4}\left(\alpha^{2}+\alpha^{-2}\right),
$$

where $\alpha=R_{g} / R_{g \text {.id }}$ is the expansion factor, with $R_{g \text {.id }}$ being the radius of gyration of the ideal chain, $R_{\text {g.id }}^{2}=N a^{2} / 6$. $F_{\text {vol }}$ refers to the volume interactions between the chain monomers, which may be written using the second and third virial coefficients as [3,31] (see the Supplemental Material [29])

$$
\beta F_{\mathrm{vol}}=\left(\frac{N^{2} B}{2 V_{g}}+\frac{N^{3} C}{6 V_{g}^{2}}\right)=\left(\frac{N^{1 / 2} \tilde{B}}{\alpha^{3}}+\frac{\tilde{C}}{\alpha^{6}}\right),
$$

where $V_{g}=(4 \pi / 3) R_{g}^{3}$ is the volume of gyration and we introduce the reduced virial coefficients, $\tilde{B}=$ $9 \sqrt{6} B /\left(4 \pi a^{3}\right)$ and $\tilde{C}=81 C /\left(4 \pi^{2} a^{6}\right)$. Finally, $F_{\text {el }}$, which takes into account all the electrostatic interactions (between the monomers and counterions) as well as the entropic part of the counterions is given by [4]

$$
\begin{aligned}
\frac{\beta F_{\mathrm{el}}}{N}= & \frac{3 \sqrt{6} \ell_{B} N^{1 / 2}(1-\tilde{\rho})^{2}}{5 \alpha}\left(1-\frac{2 R_{g}}{3 R_{0}}\right) \\
& -\frac{3}{Z}(1-\tilde{\rho}) \ln \left(\frac{R_{0}}{a}\right)-\frac{3}{2}\left(\frac{2}{\pi^{2}}\right)^{1 / 3} \frac{\ell_{B} \sqrt{6} Z^{2 / 3} \tilde{\rho}^{4 / 3}}{N^{1 / 6} \alpha} .
\end{aligned}
$$

Here, $\tilde{\rho}=\rho_{\text {in }} / \rho_{0}$ with $\rho_{\text {in }}$ being the number density of counterions within the volume occupied by the polymer chain $V_{g}$ and $\rho_{0}=N_{c} / V_{g}=N /\left(Z V_{g}\right)$ is the counterion density at the complete condensation. The value of $R_{0}$ quantifies the volume $4 \pi R_{0}^{3} / 3$ per chain in the solution and corresponds to $L$ in the MD simulations. The above expression for $F_{\text {el }}$ is valid for dilute solutions, $R_{0} \gg R_{g}$ and for $N \gg 1$ [33]. The first term in the right-hand side of Eq. (8) accounts (on the mean-field level) for the electrostatic interactions in the system, while the second term describes the entropic part of the counterion free energy. The third term quantifies the contribution from electrostatic correlations to the free energy, and is absent within the Poisson-Boltzmann approximation [4].

We now focus on the globular state where $R_{g} \sim N^{1 / 3}$, so that $\alpha \ll 1$. In this case, the entropic part of the free energy $F_{\text {id.ch }}$ [see Eq. (6)] may be ignored when compared to the other parts of the free energy $\left(F_{\mathrm{vol}}+F_{\mathrm{el}}\right)$. Also, in the collapsed regime, most of the counterions are in the vicinity of the PE, which suggests the approximation $\tilde{\rho} \approx 1$ in Eq. (8). Hence, the electrostatic contribution to the free energy can be approximated as 


$$
\frac{\beta F_{\mathrm{el}}}{N} \approx-\frac{\tilde{Z}^{2} \ell_{B}}{N^{1 / 6} \alpha}
$$

where $\tilde{Z}^{2}=(3 / 2)\left(2 / \pi^{2}\right)^{1 / 3} \sqrt{6} Z^{2 / 3}$. Thus, for a single PE in any solvent, in the regime where the electrostatic contribution to the free energy dominates over the entropic one, Eqs. (7) and (9) yield for the free energy,

$$
\frac{\beta F}{N}=-\frac{\tilde{Z}^{2} \ell_{B}}{N^{1 / 6} \alpha}+\frac{\tilde{B}}{N^{1 / 2} \alpha^{3}}+\frac{\tilde{C}}{N \alpha^{6}} .
$$

Note that while Eq. (10) takes into account the volume interactions between the chain monomers, such interactions with counterions may be also important for a dense globule. It is straightforward to take into account these interactions, which do not alter the form of the free energy (10), but lead to the renormalization of $\tilde{B}$ and $\tilde{C}$ (see the Supplemental Material [29]). For simplicity we keep the same notations for the renormalized coefficients.

In what follows we consider the case of a good solvent, which corresponds to positive coefficients $\tilde{B}$ and $\tilde{C}$. To find equilibrium $\alpha$ and hence $R_{g}$, one needs to minimize Eq. (10) with respect to $\alpha$. The relative importance of the virial terms in Eq. (10) depends on $N$, the virial coefficients $\tilde{B}$ and $\tilde{C}$, and the expansion factor $\alpha$. The second virial term dominates when $\alpha^{3}>\tilde{C} N^{-1 / 2} / \tilde{B}$, which corresponds to the weak electrostatic regime. Neglecting the third virial term in Eq. (10) and minimizing $F$ with respect to $\alpha=R_{g} / R_{g \text {.id }}$, we find

$$
R_{g}=\frac{\sqrt{\tilde{B}} a N^{1 / 3}}{\sqrt{2} \tilde{Z} \ell_{B}^{1 / 2}},
$$

as obtained in Ref. [4]. This is consistent with the MD data for $\ell_{B}<\ell_{B}^{*}: R_{g} \sim \ell_{B}^{-1 / 2}$; see Fig. 1 .

In contrast, in the strong electrostatic regime, when $\alpha^{3}<\tilde{C} N^{-1 / 2} / \tilde{B}$, the third virial term is larger than the second one. Hence, neglecting the second virial term in Eq. (10) and minimizing the free energy, we obtain

$$
R_{g}=\frac{\tilde{C}^{1 / 5} a N^{1 / 3}}{6^{3 / 10} \tilde{Z}^{2 / 5} \ell_{B}^{1 / 5}} .
$$

This scaling of $R_{g}$ is consistent with the MD simulation data for $\ell_{B}>\ell_{B}^{*}: R_{g} \sim \ell_{B}^{-1 / 5}$ as shown in Fig. 1.

To check independently our approximations for the electrostatic and the volume part of the free energy, Eqs. (9) and (7), we now calculate the respective components of the internal energy and compare them to results from MD simulations. The electrostatic part of the internal energy $E_{\mathrm{el}}=\partial\left(\beta F_{\mathrm{el}}\right) / \partial \beta$ is given by

$$
\beta E_{\mathrm{el}} /\left(N \ell_{B}\right)=-\tilde{Z}^{2} a N^{1 / 3} / \sqrt{6} R_{g} \sim N^{1 / 3} R_{g}^{-1} .
$$

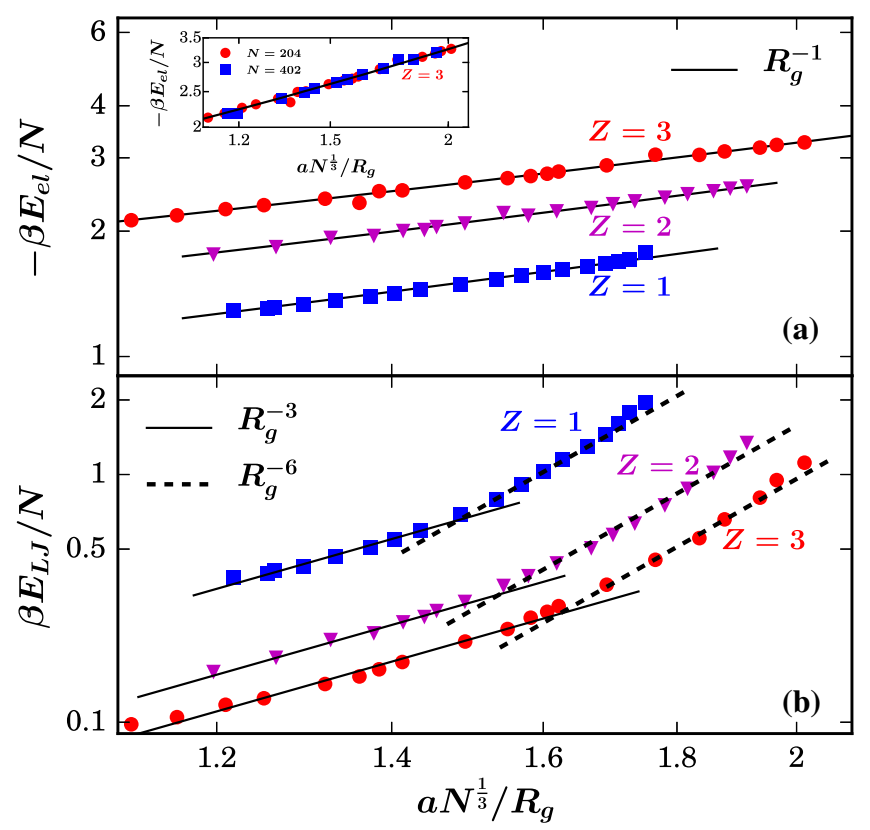

FIG. 3. (a) Variation of the electrostatic energy $E_{\mathrm{el}}$ with the radius of gyration $R_{g}$ for different valency. Inset: Variation with the chain length $N$. (b) Variation of the LJ energy of the system with the radius of gyration $R_{g}$ for different valency.

The scaling of $E_{\mathrm{el}}$ as a function of $R_{g}$ is shown in Fig. 3(a) from the MD data, which clearly demonstrates the linear dependence of the electrostatic energy $E_{\mathrm{el}}$ on the inverse gyration radius $R_{g}$ as obtained in Eq. (13). We note that this linear dependence is valid in both weak and strong electrostatic regimes.

Similarly, the internal energy corresponding to the volume interactions via $\mathrm{LJ}$ interactions, $E_{\mathrm{LJ}}=\partial\left(\beta F_{\mathrm{vol}}\right) / \partial \beta$, is given by

$$
\beta E_{\mathrm{LJ}} / N=N B^{\prime} R_{g}^{-3}+N^{2} C^{\prime} R_{g}^{-6},
$$

where $B^{\prime}=(3 / 8 \pi) \beta \partial B / \partial \beta$ and $C^{\prime}=\left(3 / 32 \pi^{2}\right) \beta \partial C / \partial \beta$. If the first term in the right-hand side of Eq. (14) dominates, one obtains $E_{\mathrm{LJ}} \sim R_{g}^{-3}$; if the second one dominates, then $E_{\mathrm{LJ}} \sim R_{g}^{-6}$. In Fig. 3(b) we plot the respective internal energy due to volume interactions from our MD data. The figure convincingly illustrates the dominance of the second and third virial terms in the weak and strong electrostatic regimes correspondingly, with the crossover occuring at $R_{g} / a N^{1 / 3} \approx 0.63(Z=3), 0.64(Z=2)$ and $0.68(Z=1)$. These values match closely with the crossover found in Fig. 1.

Conclusion.-We elucidate the origin of attractive interactions in a collapsed polyelectrolyte in a good solvent using MD simulations and theoretical analysis. We identify two collapsed regimes, that we call as weak and strong electrostatic regimes. In the first regime the gyration radius $R_{g}$ of a chain scales with Bjerrum length $\ell_{B}$ as $R_{g} \sim \ell_{B}^{-1 / 2}$ 
while in the second one as $R_{g} \sim \ell_{B}^{-1 / 5}$. This scaling is robust and independent on the valency of the counterions, volume interaction models between chain monomers and on the solvent models. Both regimes may be observed experimentally (see the Supplemental Material [29] for the discussion). The detected scaling in the weak electrostatic regime $\left(R_{g} \sim N^{1 / 3} \ell_{B}^{-1 / 2}\right)$ is not consistent with the predictions of the theories of fluctuating dipoles $\left(R_{g} \sim N^{1 / 3} \ell_{B}^{-2 / 3}\right)[23,25-27]$, or of the amorphous ionic solid $\left(R_{g} \sim N^{1 / 3} \ell_{B}^{0}\right)$ [19], but agrees with the counterionfluctuation theory [4]. At the same time the scaling in the strong electrostatic regime $\left(R_{g} \sim N^{1 / 3} \ell_{B}^{-1 / 5}\right)$ is not consistent with any of the existing theories.

In this Letter, we modified the counterion-fluctuation theory [4], in which density fluctuations of delocalized counterions inside a chain globule give rise to effective attractive interactions. Including the third virial term into the volume-interaction part of the free energy of the chain $F_{\text {vol }}$, we obtain the correct description for the $R_{g}\left(\ell_{B}\right)$ dependence in both weak and strong electrostatic regimes. We find that the different electrostatic regimes correspond to the dominance of different virial terms of $F_{\mathrm{vol}}$ and it may be envisaged that additional virial terms may be required at higher electrostatic strengths. We note that various theories explaining the origin of attractive interactions in a collapsed state of PE or PE gels $[4,19,22-27,35,36]$ differ mainly in the form of the electrostatic term. As we show in our MD simulations the scaling of the electrostatic energy with the gyration radius $R_{g}$ is the same for all values of $\ell_{B}$ and is consistent with the counterion-fluctuation theory. Hence, our results strongly support the counterion-fluctuation mechanism of the PE collapse in a good solvent, suggested previously in Ref. [4].

The simulations were carried out on the supercomputing machines Annapurna, Nandadevi, and Satpura at The Institute of Mathematical Sciences.

*anvym@imsc.res.in

†vani@imsc.res.in

*rajesh@imsc.res.in

\$nb144@leicester.ac.uk

[1] P. J. Flory, Principles of Polymer Chemistry (Cornell University, Ithaca, NY, 1953).

[2] R. R. Netz and D. Andelman, Phys. Rep. 380, 1 (2003).

[3] A. Y. Grosberg and A. R. Khokhlov, Statistical Physics of Macromolecules (AIP Press, Woodbury, NY, 1994).

[4] N. V. Brilliantov, D. V. Kuznetsov, and R. Klein, Phys. Rev. Lett. 81, 1433 (1998).

[5] A. V. Dobrynin and M. Rubinstein, Prog. Polym. Sci. 30, 1049 (2005).
[6] A. Varghese, S. Vemparala, and R. Rajesh, J. Chem. Phys. 135, 154902 (2011).

[7] G. S. Manning, J. Chem. Phys. 51, 924 (1969).

[8] A. R. Khokhlov, J. Phys. A 13, 979 (1980).

[9] M. J. Stevens and K. Kremer, Phys. Rev. Lett. 71, 2228 (1993).

[10] M. J. Stevens and K. Kremer, J. Chem. Phys. 103, 1669 (1995).

[11] R. G. Winkler, M. Gold, and P. Reineker, Phys. Rev. Lett. 80, 3731 (1998).

[12] S. M. Mel'nikov, M. O. Khan, B. Lindman, and B. Jönsson, J. Am. Chem. Soc. 121, 1130 (1999).

[13] M. Deserno and C. Holm, Mol. Phys. 100, 2941 (2002).

[14] A. A. Gavrilov, A. V. Chertovich, and E. Y. Kramarenko, Macromolecules 49, 1103 (2016).

[15] A. Schneemann, Annu. Rev. Microbiol. 60, 51 (2006).

[16] A. Siber, A. Losdorfer Bozic, and R. Podgornik, Phys. Chem. Chem. Phys. 14, 3746 (2012).

[17] R. F. Bruinsma, M. Comas-Garcia, R. F. Garmann, and A. Y. Grosberg, Phys. Rev. E 93, 032405 (2016).

[18] G. C. Wong and L. Pollack, Annu. Rev. Phys. Chem. 61, 171 (2010).

[19] F. J. Solis and O. de la Cruz, J. Chem. Phys. 112, 2030 (2000).

[20] A. Varghese, R. Rajesh, and S. Vemparala, J. Chem. Phys. 137, 234901 (2012).

[21] A. M. Tom, R. Rajesh, and S. Vemparala, J. Chem. Phys. 144, 034904 (2016).

[22] Y. Levin, Rep. Prog. Phys. 65, 1577 (2002).

[23] H. Schiessel and P. Pincus, Macromolecules 31, 7953 (1998).

[24] R. Golestanian, M. Kardar, and T. B. Liverpool, Phys. Rev. Lett. 82, 4456 (1999).

[25] A. Cherstvy, J. Phys. Chem. B 114, 5241 (2010).

[26] M. Muthukumar, J. Chem. Phys. 120, 9343 (2004).

[27] P. Kundu and A. Dua, J. Stat. Mech. (2014) P07023.

[28] $c$ is different in different theories of Refs. [23,26,27].

[29] See Supplemental Material at http://link.aps.org/ supplemental/10.1103/PhysRevLett.117.147801, which includes Ref. [12,30].

[30] J.-P. Hansen and I. R. McDonald, Theory of Simple Liquids: With Applications to Soft Matter (Academic Press, New York, 1990).

[31] Y. A. Budkov, A. L. Kolesnikov, and M. G. Kiselev, J. Chem. Phys. 143, 201102 (2015).

[32] A. Y. Grosberg and D. V. Kuznetsov, Macromolecules 25, 1970 (1992).

[33] In Ref. [4] the case of $Z=1$ has been addressed. A straightforward generalization yields Eq. (8). $R_{0}$ should be limited, since at $R_{0} \rightarrow \infty$ the entropic evaporation of counterions from a PE takes place [34].

[34] W. K. Kim and R. R. Netz, Eur. Phys. J. E 38, 120 (2015).

[35] A. R. Khokhlov and E. Y. Kramarenko, Macromolecules 29, 681 (1996).

[36] E. Y. Kramarenko, A. R. Khokhlov, and K. Yoshikawa, Macromol. Theory Simul. 9, 249 (2000). 